ARTIGO ORIGINAL ORIGINAL ARTICLE

\title{
Ansiolíticos e antidepressivos dispensados na Atenção Básica: análise de custos e interações medicamentosas
}

\author{
Anxiolytics and antidepressants drugs dispensed in \\ Primary Care: cost analysis and drug interactions \\ Clayre Anne de Araújo Aguiar¹, Felipe de Souza Macedo², Ana \\ Paula Vasconcellos Abdon ${ }^{3}$, Adriana Rolim Campos ${ }^{4}$
}

DOI: 10.21115/JBES.v8.n2.p99-107

Palavras-chave:

ansiolíticos, antidepressivos, farmacoepidemiologia,

farmacoeconomia

\section{Keywords:}

anxiolytics, antidepressants, pharmacoepidemiology, pharmacoeconomics

\section{RESUMO}

Objetivo: Identificar o quantitativo, descrever o custo dos fármacos e verificar as possíveis interações medicamentosas entre os ansiolíticos e antidepressivos dispensados pela farmácia básica dos municípios do estudo. Métodos: Estudo quantitativo, transversal e retrospectivo, realizado em municípios da região serrana cearense. A amostra foi colhida através dos livros de registros na Central de Abastecimento Farmacêutico e foram incluídos somente os medicamentos padronizados e comuns aos quatro municípios estudados, distribuídos gratuitamente à população. Resultados: Foram identificados 693 pacientes que receberam ansiolíticos e antidepressivos nos meses de janeiro e fevereiro de 2014 nos municípios. O medicamento em maior uso foi o amitriptilina, seguido pelo diazepam e fluoxetina. Apesar dos antidepressivos constituírem 54\% das unidades de medicamentos dispensadas, o seu custo correspondeu a $72 \%$ dos gastos com ansiolíticos e antidepressivos. Foi verificada a existência de interações medicamentosas importantes entre os medicamentos do estudo. Conclusões: Evidencia-se que um percentual expressivo da população estudada faz uso de psicofármacos ansiolíticos e antidepressivos, distribuição semelhante a estudos de base populacional em regiões brasileiras.
Recebido em: 25/01/2016. Aprovado para publicação em: 26/06/2016

1. Programa de Pós-Graduação em Saúde Coletiva - Universidade de Fortaleza - UNIFOR,

2. Curso de Fisioterapia - Universidade de Fortaleza - UNIFOR, Fortaleza - CE - Brasil.

3. Curso de Fisioterapia - Universidade de Fortaleza - UNIFOR, Fortaleza - CE - Brasil.

4. Programa de Pós-Graduação em Saúde Coletiva - Universidade de Fortaleza - UNIFOR, Fortaleza - CE - Brasil.

Instituição: Universidade de Fortaleza - UNIFOR.

Agradecimentos: À Fundação de Apoio ao Desenvolvimento Científico e Tecnológico (FUNCAP), à Financiadora de Estudos e Projetos (FINEP), à equipe de saúde dos municípios e as secretarias municipais de saúde.

Contatos: Clayre Anne de Araújo Aguiar, Avenida Washington Soares, 1321, Bairro Edson Queiroz, CEP 60.811-905 - Fortaleza, CE Brasil, Telefone: (85) 996773564, e-mail: clayreanne@hotmail.com 


\section{Introdução}

Os psicofármacos configuram-se como um dos recursos terapêuticos utilizados para o tratamento de pacientes com transtorno psíquico, em conjunto à escuta estabelecida na consulta médica (Brasil, 2013b). No estudo de base populacional realizado por Garcias et al. (2008), encontrou-se a prevalência de uso de $24,7 \%$ de psicofármacos nos moradores de Pelotas-RS acima de 40 anos de idade.

No que concerne à utilização de medicamentos, a Organização Mundial da Saúde (OMS) define o uso racional quando pacientes recebem os fármacos apropriados para suas condições clínicas, em doses adequadas às suas necessidades, por um período adequado e ao menor custo (Hogerzeil, 1995). Para Aquino (2008), no entanto, a realidade observada é muito diferente. Cerca de 35\% dos medicamentos são adquiridos no Brasil por automedicação. Os medicamentos são responsáveis por 27\% das intoxicações no Brasil, e 16\% dos casos de morte por intoxicações são causados por medicamentos (SINITOX, 2014). Além disso, 50\% de todos os fármacos são prescritos, dispensados ou usados inadequadamente. E o impacto financeiro também é evidente, já que os hospitais gastam em torno de $20 \%$ de seus orçamentos com as complicações causadas pela má utilização dos medicamentos (Hogerzeil, 1995).

No que se refere aos ansiolíticos e/ou antidepressivos, sabe-se que o emprego concomitante com outros medicamentos pode potencializar ou reduzir o efeito destes, daí a necessidade do conhecimento e experiência do médico prescritor. Dessa forma, foi elaborado o Protocolo de Segurança na Prescrição, Uso e Administração de Medicamentos - Ministério da Saúde - MS (Brasil, 2013a). Neste, a padronização de medicamentos nas instituições de saúde é uma das etapas preconizadas, visto que a familiaridade dos profissionais com a medicação diminui as possibilidades de erros na prescrição, dispensação e administração. Esse protocolo foi elaborado com a finalidade de promover práticas seguras no uso de medicamentos em estabelecimentos de saúde.

No que se refere à prescrição correta de medicamentos, Firmo et al. (2013) reconhecem que é uma etapa essencial para o consumo racional pelos pacientes. Assim, os medicamentos psicotrópicos devem ser prescritos apenas com receituários de controle especial (A3, B1 e B2) regulamentados pela Portaria 344/98. Para tanto, possui algumas prioridades, entre as quais está a promoção do Uso Racional de Medicamentos, que compreende a prescrição apropriada, a disponibilidade oportuna e a preços acessíveis, a dispensação em condições adequadas e o consumo nas doses indicadas, nos intervalos definidos e no período indicado de medicamentos eficazes, seguros e de qualidade (Ministério da Saúde, Portaria no 344, de 12 de maio de 1998).
Para estudo da utilização de medicamentos, faz-se necessário apontar a intoxicação exógena como um importante agravo à saúde, haja vista que os medicamentos são um dos principais causadores. Os medicamentos são responsáveis por 27\% das intoxicações no Brasil, e 16\% dos casos de morte por intoxicações são causados por medicamentos (SINITOX, 2014).

As intoxicações medicamentosas manifestam-se devido a mecanismos complexos, relacionados, dentre outros, a características individuais, à farmacodinâmica e farmacocinética, a interações medicamentosas ou com outras substâncias e ao modo de uso (Matos \& Nascimento, 2008).

No Município de Fortaleza, no ano de 2013, das 3701 intoxicações notificadas no Centro de Assistência Toxicológica (CEATOX), 284 foram causadas por medicamentos. Nos meses de janeiro e fevereiro de 2014, igual período da segunda etapa da coleta de dados desta pesquisa, foram notificadas oito intoxicações por benzodiazepínicos, cinco por antidepressivos e 22 por outros psicotrópicos (CEATOX, 2014).

Diante do elucidado, Santos (2014) aborda a "revolução psicofarmacológica" como contribuinte para a ruptura de paradigma sobre o entendimento psiquiátrico do tratamento dos transtornos mentais, já que as queixas emocionais passaram a serem vistas como produto de uma desordem neuroquímica. Logo, paulatinamente, o cenário se modificou, visto que os medicamentos passaram de coadjuvantes para protagonistas nos tratamentos psicológicos. Esta supervalorização do fármaco e a consequente medicalização do sofrimento configura-se no uso indiscriminado dos ansiolíticos e antidepressivos.

Diante do exposto, torna-se imprescindível identificar o quantitativo de ansiolíticos e antidepressivos dispensados na Atenção Básica, o impacto financeiro e as possíveis interações medicamentosas, para que políticas públicas sejam elaboradas e efetivadas com o intuito de reduzir o uso e a dependência, proporcionando-Ihes melhor qualidade de vida. Pretende-se, então, contribuir com dados epidemiológicos sobre a temática, além de sensibilizar gestores para que parcerias sejam estabelecidas a fim de formar uma equipe multiprofissional que possa auxiliar no benefício do paciente.

Com base na problemática evidenciada, o estudo tem como objetivos: Identificar o quantitativo de ansiolíticos e antidepressivos dispensados na farmácia básica da comunidade em estudo, descrever o custo dos medicamentos ansiolíticos e antidepressivos para os quatro municípios do Maciço de Baturité, e verificar as possíveis interações medicamentosas entre os ansiolíticos e antidepressivos utilizados.

\section{Metodologia}

Trata-se de um estudo quantitativo, observacional e retrospectivo. O estudo foi desenvolvido em quatro municípios de uma região serrana do Estado do Ceará, com população total 
de 38.785 habitantes em 2010 e estimativa de 39.444 habitantes em 2013 (IBGE, 2010). A população de estudo foi composta por pacientes em uso de ansiolíticos e antidepressivos, identificados em livros de registro nos meses de janeiro e fevereiro de 2014, e a coleta de dados realizada em duas etapas:

Primeira etapa: Consistiu-se no levantamento dos ansiolíticos e antidepressivos padronizados na farmácia e pactuados pelos municípios do estudo. Desta forma, identificou-se 14 (quatorze) ansiolíticos e antidepressivos pactuados e, destes, apenas 6 (seis), comuns aos quatro municípios, foram incluídos. Segunda etapa: Durante o mês de março de 2014, foram identificados os pacientes em uso de medicamentos ansiolíticos e antidepressivos no livro de registro da farmácia Central de Abastecimento Farmacêutico (CAF) da Secretaria de Saúde dos quatro municípios. Nesta etapa, foram incluídos aqueles que buscaram os medicamentos junto à farmácia (CAF) nos meses de janeiro e fevereiro de 2014, período de dois meses anteriores ao início da coleta. O tempo de dois meses foi definido para evitar receitas de retorno do paciente, pois a Portaria 344/98 estabelece que sejam dispensados medicamentos para 60 dias, no máximo (Rocha \& Werlang, 2012). A dispensação dos psicofármacos é centralizada na sede do município e o controle das receitas é feito pela farmacêutica.

Um critério de inclusão de pacientes foi buscar os fármacos comuns aos quatro municípios junto à farmácia do município nos dois meses do estudo. E foram excluídos aqueles pacientes que buscaram pela segunda vez a mesma medicação no período de análise. Os pacientes em uso de benzodiazepínicos como anticonvulsivante, como o carbamazepina, também foram excluídos.

Os dados foram registrados em planilha eletrônica: nome completo, endereço, fármaco, concentração e quantidade entregue. O cálculo das porcentagens em relação aos medicamentos utilizados foi feito com base no número total de usuários por classe farmacêutica: ansiolítico versus antidepressivo.

\section{Análise das possíveis interações medicamentosas}

A análise da potencial interação foi realizada de acordo com a base de dados do site Drugs.com e, quando não obtida a classificação da interação neste, complementou-se com o RxList. É válido assinalar que, com o objetivo de ampliar as possibilidades de interações, para esta análise permaneceram os medicamentos ansiolíticos e antidepressivos excluídos na primeira etapa, de acordo com os critérios preestabelecidos.

\section{Análise do custo dos medicamentos para os municípios}

Para análise do impacto financeiro, a quantidade de unidades distribuídas nos dois meses desta etapa foi multiplicada pelo custo da unidade farmacêutica. Obteve-se o custo do medicamento no site do Portal do Tribunal de Contas do Ceará (http://www.tce.ce.gov.br/).

A pesquisa respeita os preceitos da resolução CNS 466/12 e foi aprovada pelo Comitê de Ética em Pesquisa da Universidade de Fortaleza sob o protocolo n 520.879 .

\section{Resultados}

\section{Distribuição de ansiolíticos e antidepressivos dispensados na Atenção Básica dos Municípios estudados}

Foram identificados 693 pacientes que receberam ansiolíticos ou antidepressivos nos meses de janeiro e fevereiro de 2014 nos municípios estudados, representando 1,79\% da população em geral; além disso, 114 pacientes receberam mais de um tipo de medicamento, totalizando 807 pacientes em uso de ansiolíticos e antidepressivos. Os medicamentos mais prescritos foram, entre os antidepressivos, amitriptilina $(68,85 \%, n=316)$ e fluoxetina $(28,76 \%, n=132)$, e, entre os ansiolíticos, o diazepam $(65,23 \%, n=227)$. Estes três medicamentos representam 83,64\% ( $n=675)$ dos psicofármacos dispensados na farmácia de Atenção Básica dos municípios. Os demais fármacos distribuídos foram paroxetina, alprazolam e clonazepam (Tabela 1).

\section{Interações medicamentosas entre os fármacos dispensados}

Este estudo mostra a classificação das interações medicamentosas de acordo com a base de dados do site Drugs.com e RxList. Salienta-se que, para estes resultados, permanecem os medicamentos excluídos nas etapas de análise posterio-

Tabela 1. Quantitativo de pacientes do estudo que receberam medicamentos ansiolíticos e antidepressivos. Maciço do Baturité-CE, 2014

\begin{tabular}{|c|c|c|c|}
\hline \multirow[t]{2}{*}{$\begin{array}{l}\text { Classe } \\
\text { terapêutica }\end{array}$} & \multirow[t]{2}{*}{ Medicamentos } & \multicolumn{2}{|c|}{$\begin{array}{c}\text { Pacientes } \\
\text { em uso dos } \\
\text { medicamentos }\end{array}$} \\
\hline & & $\mathrm{n}$ & $\%$ \\
\hline \multirow[t]{4}{*}{ Antidepressivos } & Amitriptilina 25 mg & 316 & 68,85 \\
\hline & Fluoxetina 20 mg & 132 & 28,76 \\
\hline & Paroxetina 20 mg & 11 & 2,39 \\
\hline & Subtotal & 459 & 100 \\
\hline \multirow[t]{4}{*}{ Ansiolíticos } & Alprazolam 0,5 mg e 2 mg & 83 & 23,85 \\
\hline & Clonazepam 0,5 mg e 2 mg & 38 & 10,92 \\
\hline & Diazepam 5 mg & 227 & 65,23 \\
\hline & Subtotal & 348 & 100 \\
\hline Total & & 807 & \\
\hline
\end{tabular}

Fonte: Central de Abastecimento Farmacêutico dos municípios. 
res, de acordo com os critérios preestabelecidos. São eles paroxetina, venlafaxina, imipramina, nortriptilina, bupropiona, antidepressivos - não comuns aos municípios estudados - e a carbamazepina, utilizada como anticonvulsivante.

Na Tabela 2, estão as interações medicamentosas dos ansiolíticos, representados majoritariamente pelos benzodiazepínicos, e os antidepressivos, dos grupos dos antidepressivos tricíclicos, inibidores seletivos da recaptação da serotonina e noradrenalina (ISRSN), e os ISRS. Percebe-se que, das nove possíveis interações nos medicamentos prescritos, todas apontaram interação moderada.

Foi encontrado o uso concomitante de mais de um ansiolítico, conforme apresentado na Tabela 3. Para a análise da interação de dois benzodiazepínicos (BDZ), utilizou-se o RxList. Neste meio, as interações podem ser classificadas como: contraindicada, séria, significativa e menor. Desse modo, entre os BDZ, o uso tem potencial de interação significativa.

Na Tabela 4, delineia-se a interação dos distintos grupos de antidepressivos: ADT x ISRS; ADT x ISRSN; ADT x ADT. Nas quatro interações medicamentosas, três são consideradas maiores e uma moderada.

Os achados indicam também a associação entre três fármacos ansiolíticos e/ou antidepressivos, dos mais distintos

Tabela 2. Interações medicamentosas de ansiolíticos e antidepressivos classificadas por meio do site Drugs.com. Maciço do Baturité-CE, 2014

\begin{tabular}{llcl}
\hline \multirow{2}{*}{ Ansiolítico } & Antidepressivo & Frequência & $\begin{array}{c}\text { Classificação } \\
\text { de interação }\end{array}$ \\
\hline \multirow{3}{*}{ Alprazolam } & Fluoxetina & 09 & Moderada \\
\cline { 2 - 4 } & Amitriptilina & 08 & Moderada \\
\cline { 2 - 4 } & Paroxetina & 02 & Moderada \\
\hline \multirow{3}{*}{ Clonazepam } & Amitriptilina & 04 & Moderada \\
\cline { 2 - 4 } & Fluoxetina & 06 & Moderada \\
\cline { 2 - 4 } & Paroxetina & 01 & Moderada \\
\hline \multirow{3}{*}{ Diazepam } & Amitriptilina & 31 & Moderada \\
\cline { 2 - 4 } & Fluoxetina & 12 & Moderada \\
\cline { 2 - 4 } & Paroxetina & 01 & Moderada \\
\cline { 2 - 4 } & Venlafaxina & 01 & Moderada \\
\hline
\end{tabular}

Fonte: Central de Abastecimento Farmacêutico dos municípios.

Tabela 3. Interações medicamentosas classificadas por meio do site RxList entre dois ansiolíticos. Maciço do Baturité-CE, 2014

\begin{tabular}{|c|c|c|c|}
\hline \multicolumn{2}{|l|}{ Ansiolítico } & \multirow[b]{2}{*}{ Frequência } & \multirow{2}{*}{$\begin{array}{l}\text { Classificação } \\
\text { de interação }\end{array}$} \\
\hline Medicamento 1 & Medicamento 2 & & \\
\hline Diazepam & Clonazepam & 02 & Significativa \\
\hline & Alprazolam & 01 & Significativa \\
\hline
\end{tabular}

Fonte: Central de Abastecimento Farmacêutico dos municípios. grupos (Tabela 5). Dá-se ênfase à associação mais prevalente entre amitriptilina, diazepam e alprazolam.

\section{Impacto financeiro dos fármacos dispensados para os municípios}

Os medicamentos adquiridos na farmácia básica são obtidos por meio de programas do governo e distribuídos gratuitamente à população. Assim, promove despesas aos cofres públicos que, por meio de licitações, compram o medicamento de empresas fornecedoras para os municípios. Um comprimido de alprazolam custa em média $\mathrm{R} \$$ 0,47; amitriptilina, $\mathrm{R} \$ 0,57$; clonazepam, $\mathrm{R} \$ 0,50$; diazepam, $\mathrm{R} \$$ 0,30; fluoxetina, $\mathrm{R} \$ 1,42$; paroxetina, $\mathrm{R} \$ 0,56$. Assim, o Município de Aratuba, que mais consumiu amitriptilina, teria gasto $R \$ 4.708,20$ com este fármaco e $R \$ 2.163,00$ com o diazepam. Em Mulungu, os mais dispensados foram o alprazolam, com custo de $\mathrm{R} \$ 775,50$ e fluoxetina, com custo total de $\mathrm{R} \$ 3.152,40$. A paroxetina foi igualmente distribuída em Guaramiranga e Mulungu, com gasto de $\mathrm{R} \$ 84,00$ para ambos os municípios. Em Pacoti, o maior impacto financeiro foi percebido com o uso dos antidepressivos, sendo de $R \$ 1.008,90$ com amitriptilina e $R \$ 1.320,60$ com fluoxetina.

Tabela 4. Interações medicamentosas classificadas por meio do site Drugs.com entre dois antidepressivos. Maciço do Baturité-CE, 2014

\begin{tabular}{|c|c|c|c|}
\hline \multicolumn{2}{|l|}{ Antidepressivo } & \multirow[b]{2}{*}{ Frequência } & \multirow{2}{*}{$\begin{array}{l}\text { Classificação } \\
\text { de interação }\end{array}$} \\
\hline Medicamento 1 & Medicamento 2 & & \\
\hline \multirow[t]{3}{*}{ Amitriptilina } & Fluoxetina & 10 & Maior \\
\hline & Imipramina & 01 & Moderada \\
\hline & Venlafaxina & 01 & Maior \\
\hline Fluoxetina & Imipramina & 01 & Maior \\
\hline
\end{tabular}

Fonte: Central de Abastecimento Farmacêutico dos municípios.

Tabela 5. Frequência de interações medicamentosas de três fármacos das classes de ansiolíticos e antidepressivos. Maciço do Baturité-CE, 2014

\begin{tabular}{lllc}
\hline \multicolumn{2}{l}{ Ansiolítico/antidepressivo } & & \\
\cline { 1 - 3 } Medicamento 1 & Medicamento 2 & Medicamento 3 & Frequência \\
\hline Amitriptilina & Fluoxetina & Alprazolam & 01 \\
\hline Amitriptilina & Diazepam & Alprazolam & 05 \\
\hline Amitriptilina & Diazepam & Fluoxetina & 01 \\
\hline Amitriptilina & Alprazolam & Bupropiona & 01 \\
\hline Amitriptilina & Diazepam & Nortriptilina & 01 \\
\hline Amitriptilina & Diazepam & Carbamazepina & 01 \\
\hline Diazepam & Alprazolam & Fluoxetina & 01 \\
\hline Alprazolam & Clonazepam & Fluoxetina & 01 \\
\hline
\end{tabular}

Fonte: Central de Abastecimento Farmacêutico dos municípios. 
Ressalta-se que estes valores foram obtidos no site do Portal do Tribunal de Contas do Ceará, disponibilizados online, abertos à população. O cálculo de custos é referente ao primeiro bimestre de 2014.

Tomando por base o valor médio de cada comprimido nos dois primeiros meses de 2014, calcula-se a estimativa de gastos dos municípios estudados (Tabela 6).

Percebe-se que os antidepressivos são dispensados em quantidade maior do que os ansiolíticos; no entanto, a diferença entre eles, apesar de somente 2966 comprimidos, representa o custo em torno de 2,5 vezes mais do que os ansiolíticos. A fluoxetina é o medicamento de maior custo financeiro e, mesmo distribuída cerca de 3 vezes menos do que a amitriptilina, a diferença do custo total é mínima, de $\mathrm{R} \$ 7.185,20$ contra $\mathrm{R} \$ 9.260,22$ gastos com amitriptilina. A paroxetina, também pertencente à classe dos ISRs, tem custo equivalente ao da amitriptilina e é a menos prescrita.

Na classe dos ansiolíticos, as despesas somadas são intensamente representadas pelo diazepam, mesmo possuindo menor valor unitário, seguidas pelo alprazolam e clonazepam. Os benzodiazepínicos aqui descritos têm custos semeIhantes. Com isso, o impacto financeiro com os ansiolíticos e antidepressivos, nos dois meses da coleta de dados, foi estimado em $R \$ 23.269,72$, sendo a média para os dois meses, de $\mathrm{R} \$ 11.634,86$ mensais para os quatro municípios estudados do Maciço.

\section{Discussão}

Estudos que investigam o emprego de psicofármacos na Atenção Básica ou que investiguem, isoladamente, os benzodiazepínicos (Firmino et al., 2011) ou antidepressivos (Tres et al., 2013) são comumente encontrados nas regiões Sul e Sudeste do País (Rocha \& Werlang, 2012).

No Estado do Ceará, cita-se a pesquisa realizada por Passos (2008) sobre a utilização de psicofármacos em Ma- racanaú, a avaliação do consumo destes medicamentos em farmácias públicas e privadas de Pacatuba, por Silva (2009), e um estudo documental, retrospectivo e descritivo sobre a distribuição de ansiolíticos e antidepressivos no Município de Sobral (Araújo et al., 2012).

Este estudo inova por se tratar de um experimento descritivo que busca relacionar a distribuição de ansiolíticos e antidepressivos, suas potenciais interações medicamentosas e o impacto financeiro do consumo desses medicamentos em quatro municípios da Região serrana cearense.

\section{Análise da distribuição dos ansiolíticos e antidepressivos na Atenção Básica}

A análise dos resultados mostrou o maior uso de amitriptilina e diazepam dentre as classes de antidepressivos e ansiolíticos, respectivamente - dados também encontrados em pesquisa de Estudo da Utilização de Medicamentos (EUM), como a realizada em Ribeirão Preto-SP, por Sebastião \& Pelá (2004).

Em três dos quatros municípios, a maior prescrição foi de antidepressivos em relação aos ansiolíticos. A indicação dos antidepressivos como adjuvante analgésico e de emprego para dores neuropáticas, dor crônica grave, com eficácia sendo avaliada e comprovada por ensaios clínicos randomizados, pode ser justificativa para o uso elevado dessa classe de medicamentos, em especial os antidepressivos tricíclicos (ADT), como amitriptilina (Sebastião \& Pelá, 2004). No entendimento de Beltrame (2010), o aumento do consumo de antidepressivos está relacionado ao maior diagnóstico de depressão, surgimento de medicamentos e a ampliação de indicações terapêuticas a outras condições psiquiátricas.

Para Martin et al. (2012), os antidepressivos são efetivos no tratamento da depressão, mas pontua que o efeito placebo é semelhante em quadros leves. Neste estudo, predomina-se a amitriptilina em relação aos demais antidepressivos, discordando de alguns achados da literatura, na qual a fluoxetina é dispensada em maior quantidade (Rocha \& Werlang, 2012).

Tabela 6. Custo dos medicamentos ansiolíticos e antidepressivos dispensados. Maciço de Baturité-CE, 2014

\begin{tabular}{|c|c|c|c|c|}
\hline Classes terapêuticas & Medicamentos & Unidades (n) & Custo unitário ( $\mathrm{R} \$$ ) & Custo total (R\$) \\
\hline \multirow[t]{4}{*}{ Ansiolíticos } & Alprazolam & 3.430 & 0,47 & $1.612,10$ \\
\hline & Clonazepam & 1.890 & 0,50 & 945,00 \\
\hline & Diazepam & 13.440 & 0,30 & $4.032,00$ \\
\hline & Subtotal & 18.760 & & $6.589,10$ \\
\hline \multirow[t]{4}{*}{ Antidepressivos } & Amitriptilina & 16.246 & 0,57 & $9.260,22$ \\
\hline & Fluoxetina & 5.060 & 1,42 & $7.185,20$ \\
\hline & Paroxetina & 420 & 0,56 & 235,20 \\
\hline & Subtotal & 21.726 & & $16.680,62$ \\
\hline TOTAL & & 40.486 & & $23.269,72$ \\
\hline
\end{tabular}

Fonte: Central de Abastecimento Farmacêutico. Tribunal de Contas do Ceará. 
Assim, a fluoxetina está em 28,76\% ( $n=132)$ das prescrições de antidepressivos nos municípios estudados e ocupa o segundo lugar na lista de medicamentos dispensados na CAF. A fluoxetina faz parte do grupo dos ISRS e possui a menor capacidade de produzir efeitos colaterais, o que a torna um fármaco potencialmente seguro (Istilli et al., 2010). A aplicação terapêutica principal é para depressão associada à ansiedade, e este efeito benéfico no quadro ansioso contribui, segundo Morais et al. (2006), para a indicação da fluoxetina no emagrecimento. Os autores ainda ressaltam o uso irracional deste fármaco para qualquer desconforto emocional.

Além disso, como a aquisição destes medicamentos é feita gratuitamente na farmácia básica dos municípios, a prescrição médica vai ao encontro da disponibilidade do medicamento na CAF, sendo esta uma possível maneira de justificar a menor prescrição da fluoxetina em comparação à amitriptilina, o que é evidenciado também por Sebastião \& Pelá (2004) e Araújo et al. (2012). No levantamento realizado por Spagnol \& lacovski (2010), da forma de acesso da população aos psicotrópicos, 34,9\% disseram ter acesso à medicação por meio da Secretaria Municipal de Saúde. Estes resultados corroboram Castro et al. (2013), ao assinalarem que um fator contribuinte para o uso indiscriminado de psicotrópicos é a distribuição gratuita desses medicamentos por programas governamentais.

É conhecido o fato de que uma lista de medicamentos é disponibilizada pela rede própria do Programa Farmácia Popular do Brasil com um valor de dispensação mínimo, o que amplia a facilidade de acesso. Nesta rede, a dispensação dos fármacos e/ou correlatos ocorrerá mediante o ressarcimento correspondente, tão somente, aos custos de produção ou aquisição, distribuição e dispensação, conforme valores de dispensação estabelecidos (Ministério da Saúde, Portaria no 971, de 15 de maio de 2012).

A predominância do ansiolítico em relação ao antidepressivo foi observada somente em um dos quatro municípios pesquisados, comprovando estudos prévios realizados por Auchewski et al. (2004), ao dizerem que os BDZ estão entre os fármacos mais prescritos no mundo e a maior indicação é como ansiolítico e hipnótico. Ainda assim, tem-se evidenciado a redução da prescrição devido à utilização de antidepressivos para diversas indicações terapêuticas. Estudos corroboram o menor consumo dos BDZ, como o realizado em Porto Alegre - RS, no qual a prescrição de antidepressivos é maior do que o de ansiolíticos, com destaque à fluoxetina, em 28,9\% das prescrições médicas.

Quanto à classe de ansiolíticos, há o predomínio dos benzodiazepínicos e, destes, a preponderância do diazepam nos quatro municípios estudados, resultado semelhante à literatura (Araújo et al., 2012). A efetividade dos benzodiazepínicos é discutida por Castro et al. (2013) em uma revisão sistemática da literatura, na qual a análise de ensaios clínicos compro- va o uso destes para tratamento a curto prazo da ansiedade aguda e insônia e, a longo prazo, do distúrbio do pânico e da agorafobia. Os autores acrescentam que estes medicamentos, denominados também como ansiolíticos, sedativo-hipnóticos ou calmantes, têm indicação para ansiedade (associada a condições cardiovasculares ou gastrintestinais), distúrbios do sono, convulsões, espasmos musculares involuntários e dependência do álcool.

Deste modo, a diversidade de indicações terapêuticas dos benzodiazepínicos contribui para o elevado uso deste medicamento pela população. Além da diminuição da tolerância das pessoas de suportar estresse, e, assim como ocorre com os antidepressivos (Beltrame, 2010), há a introdução de novos fármacos, influência propagandística da indústria farmacêutica e prescrição inadequada por parte dos médicos.

Outra evidência que pode contribuir para o elevado uso é que, em função das altas taxas de tolerância e dependência dos BDZ, pode ocorrer o aumento da dose necessária para produzir igual efeito terapêutico e, na interrupção abrupta, promover sinais e sintomas opostos aos efeitos esperados dos fármacos (Telles Filho et al., 2011). Pesquisas indicam, também, que 50\% dos pacientes que interrompem o uso do BDZ reiniciaram o tratamento posteriormente em um ano (Huf et al., 2000).

\section{Avaliação de potenciais interações medicamentosas dos fármacos dispensados}

$\mathrm{Na}$ análise das dispensações realizadas no período do estudo referente à segunda etapa da coleta de dados, foram achadas distintas combinações de drogas para um mesmo paciente. Assim, em uma dispensação médica foi encontrada a combinação de duas classes farmacêuticas, sendo ansiolítico $x$ antidepressivo, ansiolítico $x$ ansiolítico, e antidepressivo $x$ antidepressivo; em 12 prescrições, obteve-se a prescrição de três fármacos concomitantes: dois ansiolíticos e um antidepressivo ou dois antidepressivos e um ansiolítico.

A prescrição de múltiplas medicações é uma realidade na clínica médica. No estudo de Spagnol \& lacovski (2010), dos 5946 usuários da amostra, 18\% realizaram tratamento farmacológico combinado entre ansiolíticos e antidepressivos. Assim, a associação mais frequente entre os fármacos BDZ e antidepressivos somou 1100 usuários em mais de 40 esquemas terapêuticos distintos, sendo o mais comum: diazepam e fluoxetina, diazepam e amitriptilina, e clonazepam e fluoxetina. No Maciço, predominaram o diazepam e a amitriptlina, em 31 das combinações.

No estudo de Spagnol \& lacovski (2010), a associação entre os antidepressivos e os benzodiazepínicos não foi significativa, contrapondo-se a esta pesquisa, na qual se destaca a prevalência de associação entre os antidepressivos; no entanto, nesta combinação, o predomínio foi da fluoxetina e ami- 
triptilina, em ambos os estudos, sendo que no Maciço esteve presente em dez das 13 associações entre os antidepressivos.

A terapêutica medicamentosa indicada ao transtorno depressivo sugere que, caso não haja resposta ao primeiro medicamento antidepressivo, indica-se a substituição do fármaco, aumento da dosagem ou a combinação com outra droga. O autor define que a maioria dos tratamentos para a depressão começa com um inibidor da recaptação da serotonina (ISRS). E existem interações farmacocinéticas entre ADT e ISRS, resultado da maior biodisponibilidade dos ADT e consequente exacerbação dos seus efeitos colaterais (Dunner, 2014).

Dunner (2014) aponta ainda que muitas combinações de antidepressivos são seguras, mas há dúvidas quanto à possibilidade de melhor eficácia de tais combinações. O uso concomitante de antidepressivos pode ser útil para aumentar a eficácia, mas estes são também utilizados como uma estratégia para combater os efeitos colaterais da medicação antidepressiva. Por exemplo, a trazodona é frequentemente associada com ISRS para a insônia que pode resultar do tratamento com um ISRS.

Istilli et al. (2010) assinalam que praticamente metade da amostra de seu estudo $(48,1 \%)$ em uso de antidepressivos utilizou outro medicamento associado, sendo o diazepam $(9,6 \%)$ e a amitriptilina $(5,8 \%)$ os mais utilizados. A associação entre fluoxetina e diazepam é considerada potencialmente perigosa, visto que o diazepam é substrato da enzima CYP 2C19 (citocromo P450 2C19), que promove a metabolização hepática desse composto. E a fluoxetina tende a elevar as concentrações plasmáticas do diazepam ao inibir seu metabolismo, o que poderia levar a prejuízos psicomotores e na atenção.

Por outro lado, a fase aguda da depressão é muitas vezes acompanhada de ansiedade, irritabilidade e insônia, o que pode gerar a necessidade de associar um ansiolítico, do grupo dos BDZ, ao tratamento. Os autores referem ainda que estes sintomas ansiosos podem contribuir para o abandono precoce do tratamento. Portanto, a associação entre os dois fármacos é considerada útil, em especial nas primeiras semanas de tratamento, sendo a retirada do BDZ gradual, para evitar a dependência (Kanba, 2004).

É consenso o fato de que a terapêutica medicamentosa para ansiedade e depressão abrange, em boa parte das vezes, a associação de ansiolíticos e antidepressivos (Istilli et al., 2010), corroborando os achados deste estudo, em que a maioria das associações foi entre estas duas drogas, o que pode gerar uma associação potencialmente perigosa. Nos resultados, das nove possíveis interações dos ansiolíticos com antidepressivos, todas demonstraram interação moderada, segundo a base de dados Drugs.com, o que, para Silva et al. (2010), pode resultar em exacerbação da sintomatologia do paciente, e requer, na maioria das vezes, a troca de terapia.
A interação medicamentosa da carbamazepina com os ADT, como amitriptilina e imipramina, também classificada segundo a base de dados Drugs.com em moderada, pode resultar em diminuição de 42 a 50\% dos níveis plasmáticos dos ADT. Já entre os vários ISRSs, o potencial para interações medicamentosas difere. O principal mecanismo das interações medicamentosas dos ISRSs envolve a inibição de variadas isoenzimas do citocromo P450: CYP2D6, CYP3A3/4, CYP1A2, CYP2C9/10 e CYP2C195 (Moreno et al., 1999).

De acordo com a base de conhecimentos Drugs.com, as interações medicamentosas são classificadas ainda em maiores e leves, além da moderada explicitada acima. As maiores são as que podem oferecer risco de morte e/ou requerem intervenção médica urgente, e as leves ou menores têm efeitos clínicos limitados, podendo aumentar os efeitos colaterais da terapia, mas não requerem alterações na terapêutica (Silva et al., 2010).

É importante assinalar que a interação medicamentosa mais grave, classificada como "maior", foi observada entre os antidepressivos, resultado este encontrado em 12 dos 13 pacientes que fazem uso de dois antidepressivos simultaneamente. Esse aspecto é destacado por Souza (2012), ao acentuar que o uso concomitante de dois compostos antidepressivos de mesma família ou com efeitos semelhantes sobre os neurotransmissores pode gerar a síndrome serotoninérgica, com sinais de gravidade. E, ainda mais preocupante, é a interrupção abrupta do tratamento com antidepressivos, que pode gerar ou reagudizar os pensamentos suicidas. Estes fármacos devem ser retirados gradualmente ao término do tratamento ou por ocasião de uma interrupção por motivos diversos.

O consumo de mais de um agente serotoninérgico ou o aumento na dose pode apresentar a acentuada estimulação dos receptores da serotonina nas sinapses. A sintomatologia, manifestada pela tríade de alterações clínicas no estado mental, funções motoras e autônomas, tais como confusão, hipomania, agitação, mioclonia, hiperreflexia, diaforese, diarréia, incoordenação, taquicardia e febre alta, caracteriza a síndrome serotoninérgica (Cavallazzi \& Grezesiuk, 1999). Logo, a associação entre vários fármacos pode aumentar os níveis de serotonina no organismo e, na combinação entre dois antidepressivos, o risco de síndrome serotoninérgica aumenta. Porém, também pode ocorrer na monoterapia (Dunner, 2014).

Sob este aspecto, o uso concomitante destes fármacos levanta preocupação aos pesquisadores e clínicos tanto pela possibilidade de a combinação diminuir a ação das drogas, como pela potencial toxicidade (Beltrame, 2010). Assim, a prescrição médica requer atenção criteriosa e conhecimento do medicamento, o que inclui o mecanismo de ação, fisiopatologia e monitoramento do tratamento. A observação clínica do paciente, a valorização dos efeitos colaterais e tóxicos e 
o conhecimento amplo do fármaco constituem a base para a identificação e a compreensão das interações medicamentosas (Istilli et al., 2010). Portanto, as interações dos fármacos empregados de forma concomitante devem ser bem avaliadas pelo prescritor (Souza, 2012).

No intuito de proteger a saúde coletiva, é imperativo reafirmar o papel da farmacovigilância, por ser a responsável pela avaliação da interação medicamentosa, dentre outras funções (Castro et al., 2013).

\section{Análise do impacto financeiro dos fármacos dispensados para os municípios}

De acordo com o Boletim do Sistema Nacional de Gerenciamento de Produtos Controlados (SNGPC), os ansiolíticos clonazepam, bromazepam e alprazolam foram os medicamentos controlados mais consumidos pela população do Brasil, de 2007 a 2010. No Ceará, em 2011, a ordem foi: clonazepam, alprazolam, bromazepam, amitriptilina e diazepam. O Boletim também estima os gastos das famílias brasileiras com as drogas de maior consumo. Assim, o clonazepam, primeiro da lista, pode representar um gasto de R\$ 92,4 milhões para os brasileiros. O alprazolam ocupa o terceiro lugar e a amitriptilina o quinto (Brasil, 2011).

Portanto, no condizente à saúde mental, foi criado, pela Portaria GM No 1077/00, o Programa para Aquisição dos Medicamentos Essenciais para esta área específica, financiados pela esfera federal e estadual do SUS. Nesta, foi definido que os medicamentos do programa são os constantes na Relação Nominal de Medicamentos Essenciais - RENAME (Barcelos, 2005).

Sob este aspecto, Araújo et al. (2012) destacam que os fármacos antidepressivos são a terceira classe terapêutica em termos de gastos financeiros, com representação de $4,2 \%$ do mercado farmacêutico mundial em 2002. Do total de gastos com os psicotrópicos, em Sobral, nos anos de 2010 e 2011, 16,44\% foram com ansiolíticos e antidepressivos, sendo $92,2 \%$ referentes ao custo com os antidepressivos. Tal resultado aproxima-se dos desta pesquisa, na qual os antidepressivos são responsáveis por $71,68 \%$ da receita gasta no comparativo com os benzodiazepínicos, não sendo possível contabilizar com os demais psicotrópicos, visto não ser objeto deste estudo.

Dessa forma, a amitriptilina, distribuída em maior número do que a fluoxetina, representa 55,51\% dos gastos. Já os ISRSS, mesmo dispensados em menor quantidade, têm custos próximos aos ADT, sendo a fluoxetina responsável por 43,08\% dos gastos com os antidepressivos, e a paroxetina, 1,41\%. A unidade da fluoxetina custa em média 3 vezes o valor da amitriptilina e é prescrita em menor número aos pacientes do estudo, o que pode contribuir para a justificativa do menor uso. Araújo et al. (2012) assinalam que os ADT são fármacos relativamente baratos e de fácil acesso à população.
No que se refere aos ansiolíticos, o diazepam representou $61,19 \%$ dos gastos com os BDZ, enquanto o alprazolam teve $24,47 \%$ dos custos, seguido pelo clonazepam, com 14,34\%. Estes valores discordam do estudo em Sobral, feito por Araújo et al. (2012), no qual o diazepam representou 7,66\% dos gastos com os BDZ, e o clonazepam $86,53 \%$, e com o Boletim de Farmacoepidemiologia do SNGPC, quando este afirma que o clonazepam é o ansiolítico mais comercializado no Brasil (Brasil, 2011). Ainda por Araújo et al. (2012), sabe-se que os benzodiazepínicos representam $85 \%$ das vendas de psicotrópicos, e estima-se que tenham impacto de 5,8\% no mercado mundial. No Maciço, estes fármacos representam 28,32\% da receita gasta com os ansiolíticos e antidepressivos. Ressalta-se que o comparativo com o custo referente aos demais psicotrópicos não é possível pela restrição do objeto deste estudo.

Salienta-se que os fármacos do estudo foram distribuídos gratuitamente à população, limitando a disponibilidade de outros medicamentos fora da relação dos pactuados pelos municípios e impactando nos recursos financeiros do SUS.

Convém destacar que a distribuição dos medicamentos foi proporcional aos gastos em relação às respectivas classes, contudo, em alguns medicamentos, como amitriptilina e fluoxetina, houve estreita relação (55,51\% e 43,08\%). Por fim, salienta-se que os valores por unidade farmacêutica utilizados para cálculo dos custos foram obtidos por média dos valores de dois dos quatro municípios e aplicado aos demais, sendo esta uma limitação do estudo.

\section{Conclusão}

Diante da problemática exposta, evidencia-se que um percentual expressivo da população estudada faz uso de psicofármacos ansiolíticos e antidepressivos, sendo a distribuição semelhante a estudos de base populacional em regiões brasileiras. Os resultados apontam para o maior uso de diazepam, amitriptilina e fluoxetina dentre os fármacos estudados, apesar da inserção no mercado de novos fármacos com boa resposta terapêutica e maior tolerabilidade. Apesar dos antidepressivos constituírem 54\% das unidades de medicamentos dispensadas, o seu custo correspondeu a $72 \%$ dos gastos com ansiolíticos e antidepressivos.

As potenciais interações medicamentosas encontradas, classificadas em moderada e maior na maioria das interações investigadas, demandam assistência médica e atenção rigorosa.

Com o levantamento do quantitativo de ansiolíticos e antidepressivos dispensados na farmácia básica, se desperta o interesse em realizar pesquisas que forneçam maiores informações farmacoepidemiológicas que possam subsidiar políticas públicas voltadas à Saúde Mental na Atenção Básica. Além disso, promove a sensibilização dos profissionais de 
saúde e gestores sobre o elevado uso destes medicamentos, fomentando capacitações na área de Saúde Mental.

\section{Referências bibliográficas}

Aquino DS. Porque o uso racional dos medicamentos deve ser uma prioridade? Ciências e Saúde Coletiva. 2008;13 (supl):733-736.

Araújo LL, Oliveira EN, Araújo GG, Gomes FR, Gomes BV, Rodrigues AB. Distribuição de antidepressivos e benzodiazepínicos na estratégia de saúde da família de Sobral-CE. Sanare. 2012;11(1):45-54.

Auchewski L, Andreatini R, Galduróz JC, Lacerda RB. Avaliação da orientação médica sobre os efeitos colaterais de benzodiazepínicos. Rev Brasileira de Psiquiatria. 2004; 26(1):24-31.

Barcelos RA. $O$ acesso aos medicamentos essenciais no âmbito do Sistema Único de Saúde. 2005. [Dissertação]. Porto Alegre (RS): Universidade Federal do Rio Grande do Sul; 2005.

Beltrame MM. Análise do padrão de consumo de psicofármacos: dos usuários da estratégia de saúde da família do bairro Centro, no município de Santo Ludgero - SC. 2010. [Monografia]. Criciúma (SC): Universidade do Extremo Sul Catarinense; 2010.

BRASIL. Ministério da Saúde. Agência Nacional de Vigilância Sanitária. Boletim de Farmacoepidemiologia do Sistema Nacional de Gerenciamento de Produtos Controlados. Brasilia: Ministério da Saúde, 2011.

BRASIL. Ministério da Saúde. Agência Nacional de Vigilância Sanitária. Protocolo de Segurança na Prescrição, uso e Administração de Medicamentos. Brasília: Ministério da Saúde, 2013a.

BRASII. Ministério da Saúde. Coordenação de Saúde Mental e Coordenação de Gestão da Atenção Básica. O vínculo e o diálogo necessários - inclusão das ações de saúde mental na atenção básica. Brasília: Ministério da Saúde,2013b.

Castro GLG, Mendes CMM, Pedrini ACR, Gaspar DSM, Sousa FCFS. Uso de Benzodiazepínicos como automedicação: consequências do uso abusivo, dependência, farmacovigilância e farmacoepidemiologia. Rev. Interdiscip. 2013;6(1);112-123.

Cavallazzi LO, Grezesiuk AK. Síndrome serotoninérgica associada ao uso de paroxetina: relato de caso. Arquivos de Neuro-Psiquiatria. 1999;57(3).

CEATOX. Centro de Assistência Toxicológica do Ceará. Hospital Instituto Dr José Frota. 2014.

Dunner DL . Combining antidepressants. Shanghai Archives of Psychiatry.2014; 26(6): 363-364.

Firmino KF, Abreu MH, Penini E, Magalhães SM. Fatores associados ao uso de benzodiazepínicos no serviço municipal de saúde da cidade de Coronel Fabriciano, Minas Gerais, Brasil. Cad. Saúde Pública. 2011; 27(6).

Firmo WC, Paredes AO, Cunha CL, Torres AG, Buccini DF. Análise das prescrições médicas de psicotrópicos de uma farmácia comercial no município de Bacabal, Maranhão. J. Manag. Prim. Health Care, 2013; 4(1):10-18

Garcias CMM, Pinheiro RT, Garcias GL, Horta BL, Brum CB. Prevalência e fatores associados ao uso de antidepressivos em adultos de área urbana de Pelotas, Rio Grande do Sul, Brasil, em 2006. Cad. Saúde Pública. 2008;24(7):1565-1571.

Hogerzeil HV. Promoting Rational Prescribing: An International Perspective. British Journal of Clinical Pharmacology. 1995; 39:1-6.

Huf G, Lopes CS, Rozenfeld S. O uso prolongado de benzodiazepínicos em mulheres de um centro de convivência para idosos. Cad. Saúde Pública. 2000;16(2).
IBGE. Instituto Brasileiro de Geografia e Estatística. Censo 2010. Disponível em: <http://www.ibge.gov.br>.

Istilli PT, Miasso Al, Padovan CM, Crippa JA, Tirapelli CR. Antidepressivos: uso e conhecimento entre estudantes de enfermagem. Rev. Latino-am. Enfermagem. 2010;18(3):131-139.

Kanba S. Although antidepressants and anxiolytics are frequently used together to treat depression in the acute phase, how effective is the concomitant use of these drugs? Journal of Psychiatry and Neuroscience. 2004; 29(6): 485.

Matos GC, Nascimento AC. Impacto dos medicamentos como agentes de intoxicações humanas. Revista Racine. 2008;106(5): 59-66.

Martin D, Cacozzi A, Macedo T, Andreoli SB. Significado da busca de tratamento por mulheres com transtorno depressivo atendidas em serviço de saúde pública. Interface. 2012;16(43):885-899.

Ministério da Saúde. Portaria no 344, de 12 de maio de 1998. Aprova o Regulamento Técnico sobre substâncias e medicamentos sujeitos a controle especial. [Acessado 2015 ago 14]. Disponível em: http://www. anvisa.gov.br/hotsite/talidomida/legis/Portaria_344_98.pdf.

Ministério da Saúde. Portaria no 971, de 15 de maio de 2012. Dispõe sobre o Programa Farmácia Popular do Brasil. [Acessado 2015 ago 14]. Disponivel em: http://bvsms.saude.gov.br/bvs/saudelegis/gm/2012/ prt0971_15_05_2012.html.

Morais EC, Pissatto S, Prado JN, Foppa T, Murakami FS, Silva M.A. Análise da prescrição e consumo de cloridrato de fluoxetina no município de Florianópolis (SC). Revista Saúde e Ambiente. 2006; 7(2):7-11.

Moreno RA, Moreno RH, Soares MB. Psicofarmacologia de antidepressivos. Revista Brasileira de Psiquiatria. 1999;21(supl.1):24-40.

Passos AC. Utilização de psicofármacos entre usuários da atenção primária do município de Maracanaú-Ceará. 2008. [Dissertação]. Fortaleza (CE): Universidade Federal do Ceará; 2008.

Rocha BS, Werlang MC. Psicofármacos na Estratégia Saúde da Família: perfil de utilização, acesso e estratégias para a promoção do uso racional. Rev. Ciência \& Saúde Coletiva. 2012; 8(11):3291-3300.

Santos KY. Uma análise dos efeitos do uso a longo prazo de antidepressivos. 2014. [Dissertação]. Assis: Universidade Estadual Paulista; 2014.

Sebastião ECO, Pelá IR. Consumo de psicotrópicos: análise das prescrições ambulatoriais como base para estudos de problemas relacionados com medicamentos. Seguim. Farmacoter. 2004;2(4):250-266.

Silva DM. Avaliação do consumo de medicamentos psicotrópicos no município de Pacatuba. 2009. [Monografia]. Fortaleza(CE): Escola de saúde pública do Ceará; 2009

Silva NM, Carvalho RP, Bernardes AC, Moriel P, Mazzola PG, Franchini CC. Avaliação de potenciais interações medicamentosas em prescrições de pacientes internadas, em hospital público universitário especializado em saúde da mulher, em Campinas-SP. Revista de Ciências Farmacêuticas Básica e Aplicada. 2010; 31(2): 171-176.

SINITOX. Sistema Nacional de Informações Tóxico-Farmacológicas. 2014. [Acessado 2014 nov 22]. Disponível em: sinitox http://www.fiocruz.br/ sinitox/media/med\%20tabela\%201.pdf.

Souza CA. Uso racional de antidepressivos. Pol+Br. 2012;17(6).

Spagnol WP, lacovski RB. Uso de medicamentos psicotrópicos no Programa Saúde Mental no Município de Água Doce-SC. Ágora. R. Divulg. Cient. 2010; 17(1):94-102.

Telles Filho PC, Chagas AR, Pinheiro ML, Lima AM, Durão AM. Utilização de benzodiazepínicos por idosos de uma estratégia de saúde da família: implicações para enfermagem. Esc Anna Nery. 2011;15(3):581-586.

Tres J, Antoniolli MA, Anzolin V, Ferraz L. Perfil dos usuários de medicamentos antidepressivos e a assistência de uma farmácia pública. Biofar, Rev. Biol. Farm. 2013; 9(2): 80-88. 